\title{
Statistical Analysis of Optimal Energy and Security Controls Under Different Sources of Uncertainties
}

\author{
Omid Alizadeh Mousavi, Rachid Cherkaoui, Mokhtar Bozorg \\ Power Systems Group \\ École Polytechnique Fédérale de Lausanne (EPFL) \\ Lausanne, Switzerland \\ omid.alizadeh@epfl.ch, rachid.cherkaoui@epfl.ch,mokhtar.bozorg@epfl.ch
}

\begin{abstract}
The management of uncertainties is a challenging task for reliable and secure operation of power systems. The uncertainties come from multiple sources, including the forecast errors of wind power and load, the forced outage of generating units, loss of transmission equipments, etc. This paper classifies different uncertainties based on their binary and continuous attributes. The main idea is to investigate the effect of each source of uncertainties on the cost of energy and security controls. For this purpose, a specific optimization method is developed which takes into account a forecasted scenario and a stochastic scenario. This optimization problem is solved for a fixed forecasted scenario and a varying stochastic scenario. The stochastic scenarios are constructed using a Monte Carlo Simulation that considers various sources of uncertainties. The main advantage of the proposed optimization is that the number of incorporated stochastic scenarios does not increase the size of the optimization problem. The models of different uncertainties, particularly wind power forecast errors, are discussed in depth. This optimization allows obtaining the statistical moments and constructing the probability distributions. The proposed optimization approach is then applied to the IEEE RTS 24-bus system. The probability distributions and statistical moments of objective functions and control variables are assessed for three cases, namely: (i) with only binary uncertainties, (ii) with only continuous uncertainties and (iii) with both of them.
\end{abstract}

Keywords - binary and continuous uncertainties, wind power forecast errors, Monte Carlo simulation, heavy-tailed distributions.

\section{INTRODUCTION}

The power grids are continuously exposed to numerous sources of uncertainties which threaten the reliable and secure electricity supply to the customers. These uncertainties mainly arise from the occurrence of contingencies as well as the forecast deviations of load and more recently renewable energy production. The growing share of intermittent renewable generation capacity will increase more and more the uncertainties in power systems. In the liberalized electricity market environment, the concept of ancillary services is introduced to guarantee the secure operation of power system under different uncertainties. The frequency control reserve is among the most prominent ancillary services for secure operation of the system. The reserve can be scheduled in a joint energy/reserve market in order to avoid the market inefficiencies of separate and sequential markets [1]. In this respect, the cost of system operation can be represented in terms of the cost of providing energy and security.
The management of uncertainties in power systems regarding the system security has received many attentions from power engineering communities using deterministic (e.g. $\mathrm{N}-1$ criteria) and probabilistic (e.g. stochastic and robust) optimization approaches [2]. These optimization methods aim to obtain a balance between the economy and security. In general, the optimization problems for the management of resources characterize the different uncertainties by taking into account a certain number of scenarios [3]. These scenarios are selected from all possible scenarios using different scenario generation/reduction approaches. On one hand, the consideration of all scenarios is not plausible since the computational burden increases drastically and the obtained solution becomes very expensive from the economical point of view. On the other hand, when a power system is designed to be robust to a specific class of perturbations, it becomes more vulnerable to another class of failures [4]. These issues become even more challenging for consideration of the lowprobability/high-impact scenarios [5]. Therefore, the optimized solutions as well as the evaluated value of risk, as the representative of the security cost, are dependent on the selected scenarios.

The probability distributions of the optimal solutions for all scenarios can reveal important information about the impact of uncertainties on the management of resources. In order to obtain the probability distributions and get rid of the abovementioned scenario related problems, this paper proposes an optimization approach based on Monte Carlo simulation (MCS). In this respect, a joint energy/reserve market is assumed. This optimization consists of two system states including a forecasted state and another scenario related to uncertainties. Different sources of uncertainties are constructed using a MCS approach. For every MCS scenario, the proposed optimization is carried out for the combination of the forecasted state and a stochastic scenario. The optimization is repeated for a number of scenarios in order to obtain the required accuracy level for the statistical moments of obtained solutions. In order to model the uncertainties, this paper firstly classifies them according to their binary and continuous attributes (see section II). The obtained solutions are useful to evaluate the effect of different sources of uncertainties on the optimal energy and security controls. The peculiarities of these probability distributions, like type and statistical moments, are further investigated. 
The structure of the paper is the following. Section II describes the simulation approach including the applied MCS method and the models of different uncertainties like forecast errors of wind power and load as well as equipment outages. The detailed formulation of the proposed optimization is given in section III. Section IV presents the simulation results with reference to IEEE RTS 24-bus system. The probability distributions and the statistical moments of optimal solutions are investigated in depth. Finally, the conclusions are discussed in section $\mathrm{V}$.

\section{ANALYSIS OF PROPOSED APPROACH}

The proposed optimization approach minimizes the total cost of energy and security regarding different sources of uncertainties. Several security actions are taken into consideration including upward/downward reserves $\left(R_{G}^{u p}\right.$ and $\left.R_{G}^{d n}\right)$, wind power spillage $\left(P_{W}^{\text {spil }}\right)$, generating unit shut down and load shedding $\left(P_{D}^{l s d}\right)$. This optimization includes two system states. The first one represents a given forecasted state and another state characterizes a stochastic scenario. The stochastic scenario state represents the uncertainties. It is constructed using a MCS considering different sources of uncertainties. The forecasted state is determined beforehand and remains fixed for all scenarios. In this way only a stochastic scenario is considered for each optimization. The advantage of this approach is that the number of considered scenarios is not limiting anymore since it does not increase the size of optimization problem.

Furthermore, the MCS is an effective approach to include the uncertainties in the system management. It relies on the deterministic model simulation for a necessary number of model evaluations. For every evaluation of MCS a sample from the uncertain space is taken. The different uncertainties are classified into binary and continuous attributes categories. The binary uncertainties include the outage of transmission elements and generating units. The continuous uncertainties take into account the forecast errors of the load and renewables' power output. Here, only wind power production is studied as renewable generation. The detailed modelling of these uncertainties is carefully revisited below.

The state of each component with binary uncertainties is derived by sampling based on its own availability. Moreover, the dagger sampling is used as a variance reduction technique to improve the performance of the MCS [6]. This method is appropriate for binary variables and small probability events. In this sampling method, for each component with failure probability $p$, a single failure is randomly selected within each $1 / p$ trials. Hence, only one random number determines the state of the component for $1 / p$ trials.

Regarding the load forecast errors several studies have demonstrated that it follows a normal distribution. This distribution is described by the first two statistical moments: mean and variance. The mean value $(\mu)$ is equal to the amount of load $\left(P_{d}\right)$ and a $5 \%$ of the variance $(\sigma)$ is also assumed.

Many studies assume that the wind power forecast errors follow a normal distribution. However, this is a simplistic assumption since the third and fourth statistical moments of this distribution have none-zero values [7]. The consideration of these moments, namely skewness and kurtosis, allows analyzing more realistic scenarios. The skewness $(\gamma)$ is a measure of probability distribution's asymmetry and the kurtosis $(\kappa)$ describes the magnitude of distribution's peak. Other distributions have been investigated for the wind power forecast errors, including Weibull [8], beta [9] and hyperbolic [7] distributions. In order to get rid of the selection of a particular distribution, the wind power forecast errors is modeled using Pearson system random numbers [10]. In this approach, the four statistical moments are given and the random wind power outputs are generated in such a way to fulfill these statistical moments. It is worth noting that these moments can be normalized based on wind power capacity.

The presented models for uncertainties are incorporated in the proposed optimization based on MCS. The output of the MCS based optimization is the probability distributions and four statistical moments of the objective function and control variables. Hence, the stopping criterion for MCS is defined as the maximum deviation of the estimated moments between two iterations. The flowchart of the proposed optimization approach is presented in Figure 1. The detailed formulation of the optimization problem at each iteration and the stopping criterion are described in section III.

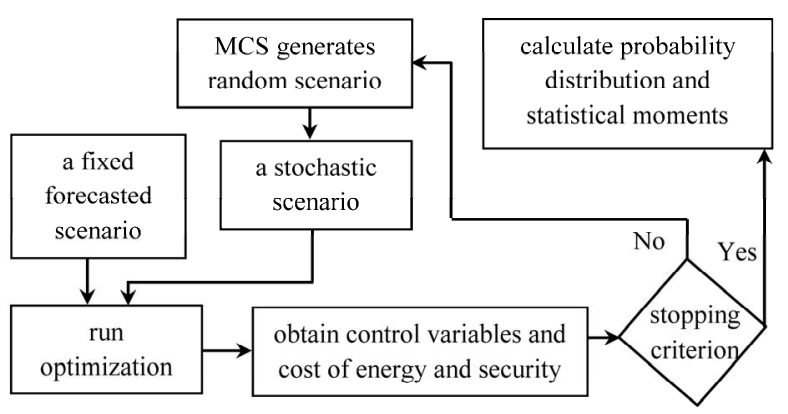

Fig. 1. Flowchart of proposed optimization approach.

\section{PROBLEM FORMULATION AND SOLUTION}

The proposed optimization for every iteration of the MCS is presented in details. Each scenario includes the availability of generators $\left(\bar{u}_{g}^{s}\right)$ and transmission elements $\left(\bar{u}_{l}^{S}\right)$ as well as the amount of load $\left(\bar{P}_{D}^{S}\right)$ and wind power output $\left(\bar{P}_{W}^{S}\right) . \bar{u}_{g}^{S}$ and $\bar{u}_{l}^{S}$ represent the binary uncertainties where 0 indicates the outage of element and 1 otherwise. The state of binary uncertainties is obtained based on their given availability and using the dagger sampling method as explained in section II. The grid is represented based on the DC load flow model. It is worth noting that the topology dependent matrices, such as the imaginary part of the nodal admittance matrix $\left(B_{b u s}\right)$ and the incidence matrix considering the serial susceptance of network branches $\left(B_{f}\right)$, change for the scenarios where $\bar{u}_{l}^{s}$ includes components equal to 0 . Hence, for every scenario these two matrices are shown by $B_{b u s}^{S}$ and $B_{f}^{S}$. Moreover, $\bar{P}_{D}^{s}$ and $\bar{P}_{W}^{S}$ represent the continuous uncertainties. For every scenario, the load forecast errors is modeled using normal distribution, whereas, the wind power forecast errors is modeled using the four statistical moments. 
Under the abovementioned assumptions, the optimization formulation is presented by (1)-(14). In this formulation, the constant parameters are shown with over bar. The superscript " $s$ " indicates the variables and parameters in the related stochastic scenario state. The objective function, given by (1), is the minimization of the cost of energy and security control actions. It is subjected to the constraints at the forecasted state, given by (2)-(5), and also at the scenario state, given by (6)(14).

$$
\begin{aligned}
\min \sum_{g \in \Omega_{G}}\left(P_{G g} \cdot \bar{C}_{g}^{a} \cdot P_{G g}\right. & \left.+\bar{C}_{g}^{b} \cdot P_{G g}+\bar{C}_{g}^{c} \cdot u_{g}\right)+\sum_{g \in \Omega_{G}} \bar{C}_{r}^{u p} \cdot R_{G g}^{u p} \\
& +\sum_{g \in \Omega_{G}} \bar{C}_{r}^{d n} \cdot R_{G g}^{d n}+\sum_{w \in \Omega_{w}} \bar{C}_{w}^{s p i l} \cdot P_{W w}^{s p i l} \\
& +\sum_{d \in \Omega_{D}} \bar{C}_{d}^{l s d} \cdot P_{D d}^{l s d}+\sum_{g \in \Omega_{G}} \bar{C}_{g}^{s d} \cdot u_{g} \cdot\left(1-\bar{u}_{g}^{s}\right)
\end{aligned}
$$

subject to

$$
\begin{array}{cr}
u_{g} \cdot \bar{P}_{G g}^{\min } \leq P_{G g} \leq u_{g} \cdot \bar{P}_{G g}^{\max } & \forall g, g \in \Omega_{G} \\
\left|\bar{B}_{f} \cdot \theta_{i}\right| \leq \bar{P}_{l}^{\max } & \forall l, l \in \Omega_{T} \\
P_{G i}+\bar{P}_{W i}-\bar{P}_{D i}=\bar{B}_{b u s} \cdot \theta_{i} & \forall i, i \in \Omega_{B} \\
\sum_{i \in \Omega_{B}}\left(P_{G i}+\bar{P}_{W i}-\bar{P}_{D i}\right)=0 & \\
u_{g} \cdot \bar{P}_{G g}^{\min } \cdot \bar{u}_{g}^{s} \leq P_{G g}^{s}+R_{G g}^{u p}-R_{G g}^{d n} \leq u_{g} \cdot \bar{P}_{G g}^{\max } \cdot \bar{u}_{g}^{s} & \forall g, g \in \Omega_{G} \\
\left|\bar{B}_{f}^{s} \cdot \theta_{i}^{s}\right| \leq \bar{P}_{l}^{\max } & \forall l, l \in \Omega_{T} \\
0 \leq R_{G g}^{u p} & \forall g, g \in \Omega_{G} \\
0 \leq R_{G g}^{d n} & \forall g, g \in \Omega_{G} \\
0 \leq P_{D d}^{l s d} \leq \bar{P}_{D d}^{s} & \forall d, d \in \Omega_{D} \\
0 \leq P_{W w}^{s p i l} \leq \bar{P}_{W w}^{s} & \forall w, w \in \Omega_{W} \\
P_{G i}^{s}+R_{G i}^{u p}-R_{G i}^{d n}+\bar{P}_{W i}^{s}-P_{W i}^{s w i l}-\bar{P}_{D i}^{s}+P_{D i}^{l s d}=\bar{B}_{b u s}^{s} . \theta_{i}^{s} & \forall i, i \in \Omega_{B} \\
\sum_{i \in \Omega_{B}}\left(P_{G i}^{s}+R_{G i}^{u p}-R_{G i}^{d n}+\bar{P}_{W i}^{s}-P_{W i}^{s p i l}-\bar{P}_{D i}^{s}+P_{D i}^{l s d}\right)=0 & \\
P_{G g}^{s}=P_{G g} & \forall g,\left\{g \in \Omega_{G} \mid \bar{u}_{g}^{s}=1\right\}
\end{array}
$$

The price vectors of upward/downward reserves are given by $\bar{C}_{r}^{u p}$ and $\bar{C}_{r}^{d n}$, respectively. The price vectors of generation are given by $\bar{C}_{g}^{a}, \bar{C}_{g}^{b}, \bar{C}_{g}^{c}$. The prices of wind power spillage, load shedding and generating unit shut down are given by $\bar{C}_{w}^{\text {spil }}, \bar{C}_{d}^{\text {lsd }}$ and $\bar{C}_{g}^{s d}$, respectively. $\Omega_{G}, \Omega_{D}, \Omega_{T}, \Omega_{W}$ and $\Omega_{B}$ (resp. $g, d, l, w, i$ ) are the set (resp. index) of generators, demands, transmission elements, wind generations and buses, respectively. The voltage angle of buses at the forecasted state and at stochastic scenario state are shown by $\theta_{i}$ and $\theta_{i}^{s}$, respectively.

The first term of the objective function is the quadratic energy cost of generating units. The second and third terms represent the cost of upward and downward reserves, respectively. The fourth, fifth and sixth terms are the cost of wind spillage, load shedding and generating unit shut down, respectively. The cost of generating unit shut down is taken into consideration whenever a generating unit is committed at the forecasted states $\left(u_{g}=1\right)$ and it goes out at the stochastic scenario state $\left(\bar{u}_{g}^{s}=0\right)$.

The constraints on lower/upper limits of generating units power output, shown by $\bar{P}_{G}^{\min }$ and $\bar{P}_{G}^{\max }$, at the forecasted scenario (resp. at the stochastic scenario) is given by (2) (resp. (6)). The constraint (3) (resp. (7)) ensures that the power flow of lines is below of transmission capacity $\left(\bar{P}_{l}^{\max }\right)$. The power flow equation is presented by (4) (resp. (12)). The generation and demand power balance equation is given by (5) (resp. (13)). It is assumed that generating units can offer the possible amount of reserve bounded to their $\mathrm{min} / \mathrm{max}$ generating power output. Thus, the constraints on the amount of reserves are given by (6), (8) and (9). The amounts of load shedding and wind power spillage are limited by (10) and (11), respectively. The equation (14) guarantees that the power output of generating units are equal at the forecasted and stochastic state. This is valid only for those generating units that are available at both operating points.

The proposed optimization is a quadratic programming with mixed-integer linear constraints. The optimization problems are solved using the solver Gurobi [11] via the MATLAB interface YALMIP [12].

The optimization results for every scenario are the generating units power output $\left(P_{G}\right)$ and its on/off status $\left(u_{g}\right)$, upward/downward reserve $\left(R_{G}^{u p}, R_{G}^{d n}\right)$, wind power spillage $\left(P_{W}^{s p i l}\right)$ and load shedding $\left(P_{D}^{l s d}\right)$. The probability distribution and statistical moments $(\mu, \sigma, \gamma, \kappa)$ of the simulation results are investigated for a given case study in section IV. For this purpose, the stopping criterion of MCS is defined as the maximum deviation of the estimated moments between two iterations. Therefore, the stopping criterion at $k$-th iteration of MCS is shown by $z_{k}$ and given as follows:

$$
z_{k}=\max \left\{\frac{\mu_{k}-\mu_{k-1}}{\mu_{k}}, \frac{\sigma_{k}-\sigma_{k-1}}{\sigma_{k}}, \frac{\gamma_{k}-\gamma_{k-1}}{\gamma_{k}}, \frac{\kappa_{k}-\kappa_{k-1}}{\kappa_{k}}\right\} \leq \epsilon
$$

\begin{tabular}{|c|c|c|c|c|c|c|c|}
\hline \multirow{2}{*}{ Name } & \multirow{2}{*}{ Bus } & \multirow{2}{*}{$\begin{array}{l}\text { Forecasted Power Output } \\
\text { (MW) }\end{array}$} & \multirow{2}{*}{$\begin{array}{c}\text { Installed Capacity } \\
\text { (MW) }\end{array}$} & \multicolumn{4}{|c|}{ Statistical Moments of Forecast Errors } \\
\hline & & & & $\mu$ & $\sigma$ & $\gamma$ & $\boldsymbol{\kappa}$ \\
\hline WP1 & 17 & 100 & 240 & -0.0123 & 0.0827 & 0.3063 & 3.0311 \\
\hline WP2 & 21 & 180 & 340 & 0.0092 & 0.0450 & -0.2891 & 3.5896 \\
\hline WP3 & 22 & 50 & 100 & -0.0005 & 0.0534 & 0.1378 & 2.3859 \\
\hline
\end{tabular}

where $\epsilon$ is the desired error level. The smaller is $\epsilon$, the higher is the required number of MCSs. Here, the stopping criterion is defined for the total cost of the system.

\section{Simulation Results}

The proposed optimization approach is evaluated on the IEEE RTS 24-bus system in order to study the effect of different sources of uncertainties on the energy and security management. The single line diagram of the system is shown in Figure 2. Its description and data, including reliability information, can be found in [13]. The total amount of load and generation capacity are equal to $2850 \mathrm{MW}$ and $3405 \mathrm{MW}$, respectively. Three wind power generations are added to the system according to the given data in Table I. Here, the wind penetration level is equal to $20 \%$. It is worth noting that the

TABLE I.

DATA OF WIND POWER GENERATIONS. 


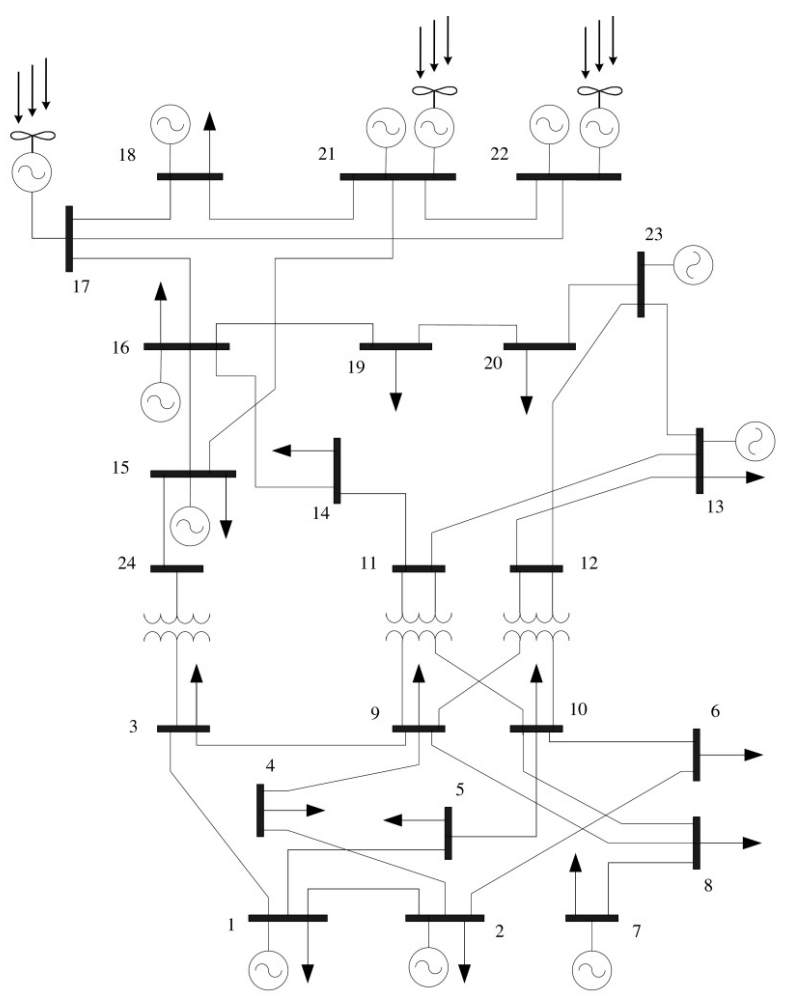

Fig. 2. Single line diagram of IEEE RTS 24-bus system.

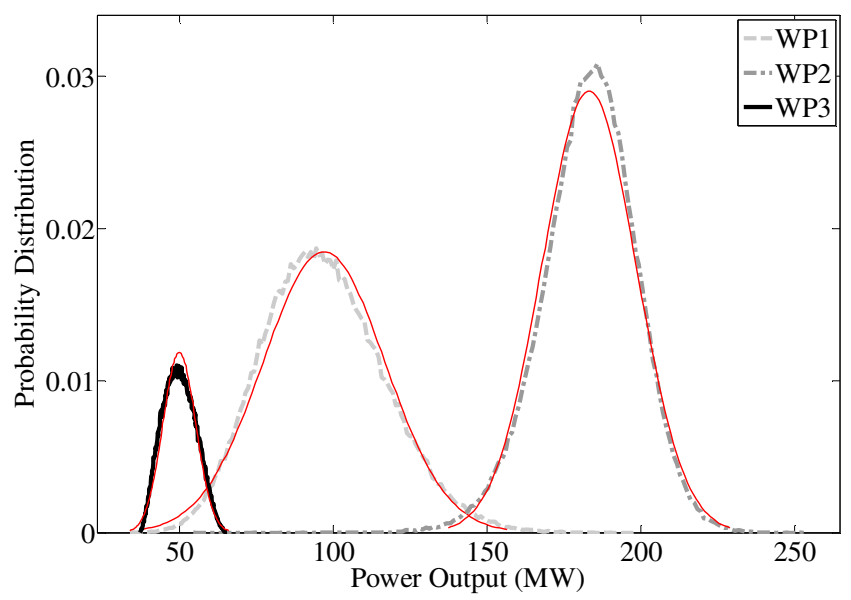

Fig. 3. Probability distributions of wind power forecast errors and their normal distribution fits in red for three wind generations. normalized based on wind power capacity. The normalized statistical moments are taken from the day-ahead wind power forecast errors of several systems, including Ireland, Germany and Denmark, as reported in [7]. The probability distributions of wind power forecast errors and their normal distribution fits in red are illustrated in Figure 3. Moreover, 5\% load forecast errors is also taken into consideration. Here, the prices of upward/downward reserves are assumed equal to $20 \%$ of $\bar{C}_{g}^{b}$. The prices of wind spillage and load shedding are assumed equal to $100 \$ / \mathrm{MW}$ and $1000 \$ / \mathrm{MW}$, respectively.

In this paper three cases are studied. The first case, shown by "Case A", only assumes the continuous uncertainties, whereas, the second case, shown by "Case B", only considers the binary uncertainties. The third case, shown by "Case C", addresses both of binary and continuous uncertainties.

The cumulative distributions of energy and security costs are shown in Figure 4 and Figure 5, respectively. The results are plotted in log-linear axes in order to effectively demonstrate the low probability regions of distributions. It is interestingly observed that the distribution of Case $\mathrm{C}$ for energy cost below $1344 \$$ (resp. security cost below $18.17 \$$ ) is similar to the distribution of Case A, whereas, for larger amounts of energy cost (resp. security cost) it follows the distribution of Case B. This observation demonstrates that for the lower costs with higher probabilities the effect of continuous uncertainties is more important, while for the higher costs with lower probabilities the effect of binary uncertainties becomes more important.

This conclusion can be also deduced from the probability of scenarios with the energy cost higher than the cost in the case with no stochastic scenario (resp. the probability of scenarios with non-zero security cost). These probabilities show the number of scenarios with the abovementioned characteristics per total number of scenarios. These values for different cases are given in Table II. The lowest probabilities are observed for Case B. It shows that additional costs due to binary uncertainties are less probable. However, as demonstrated in Figure 4 and Figure 5, for these low probable cases the amounts of additional costs are much higher. On the contrary, the continuous uncertainties impose lower amounts of additional costs but with higher frequency. Therefore, higher amount of security actions is necessary with a lower probability for the binary uncertainties, whereas, lower amount of security actions is required with a higher probability for the continuous uncertainties.

In addition, several types of well-known distributions are tested to fit the distribution of the obtained results. The generalized Pareto distribution and the generalized extreme value distribution are the most appropriate for fitting the

TABLE II. PROBABILITY OF SCENARIOS WITH THE ENERGY COST HIGHER THAN THE COST IN THE CASE WITH NO STOCHASTIC SCENARIO PROBABILITY OF SCENARIOS WITH NON-ZERO SECURITY COST.

\begin{tabular}{lcc}
\hline & \multicolumn{2}{c}{ probability } \\
\cline { 2 - 3 } & energy cost & security cost \\
\hline Only Continuous (Case A) & 0.1433 & 0.5589 \\
Only Binary (Case B) & 0.0199 & 0.0195 \\
Both of them(Case C) & 0.1614 & 0.5659 \\
\hline
\end{tabular}




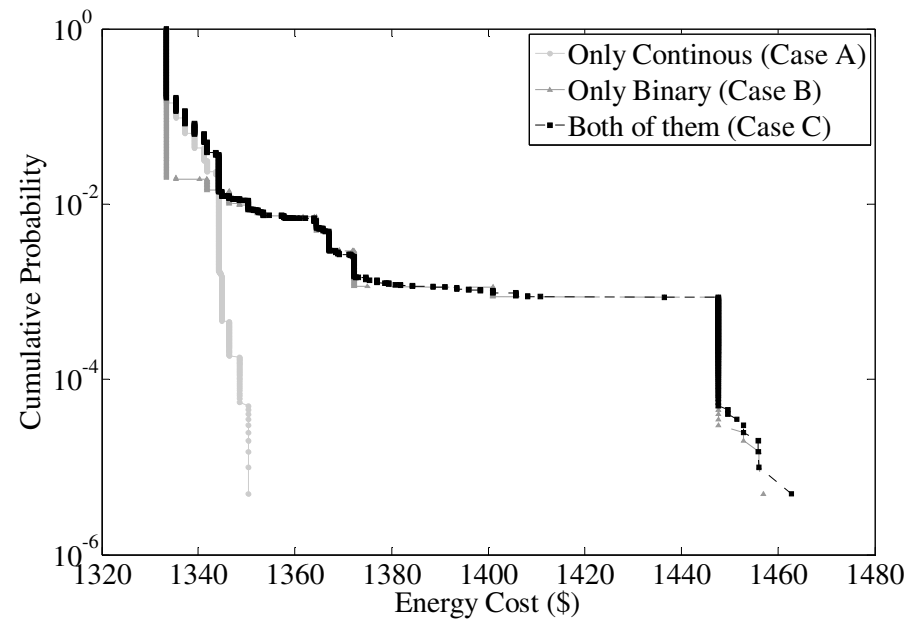

Fig. 4. Cumulative probability distribution of energy cost for all cases.

obtained results. These heavy-tailed families of distributions indicate that the occurrence of extreme values cannot be overlooked. Moreover, in these distributions the third and fourth statistical moments are non-zero in general.

In this respect, the statistical moments of the energy and security costs are given in Table III. The expected cost $(\mu)$ and the standard deviation $(\sigma)$ are higher for Case $\mathrm{C}$ in comparison with other cases, since both of the binary and continuous uncertainties are taken into consideration. The highest values of skewness $(\gamma)$ and kurtosis $(\kappa)$ for Case B show that the effect of binary uncertainties are much higher on the asymmetry and the peak magnitude of distributions. As a result, the impact of binary uncertainties is more significant on the heavy tail behavior of distributions.

The distribution of several control variables demonstrates similar behavior as that of the energy and security costs distributions. These control variables include the generating unit output power at buses $7,13,15,23$, the upward reserve at buses $7,15,23$, and the downward reserve at buses18, 22. For instance, the cumulative distributions of the generating unit

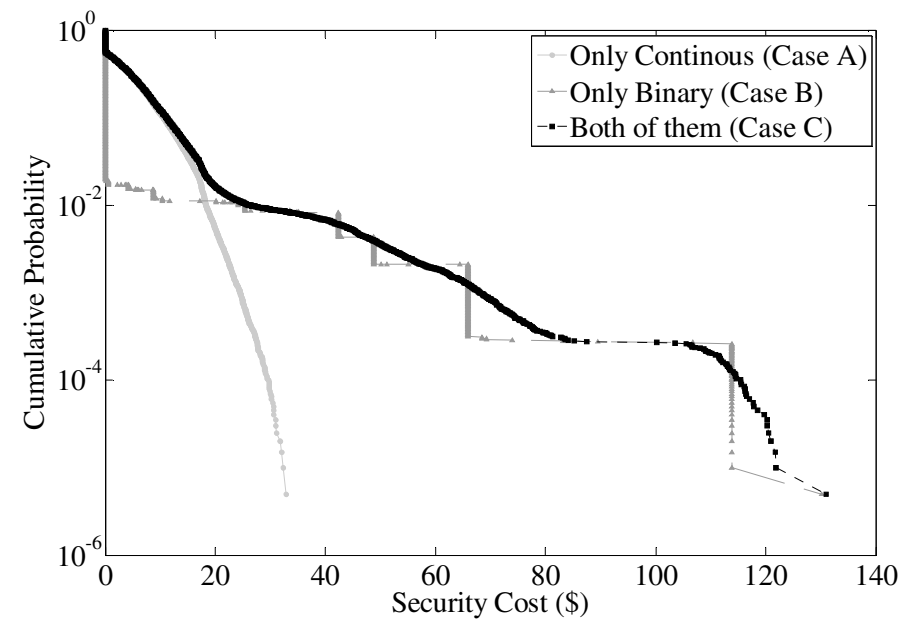

Fig. 5. Cumulative probability distribution of security cost for all cases.

output power at bus 15 , the upward reserve at bus 13 and the downward reserve at bus 22 for all cases are illustrated in Figure 6, Figure 7 and Figure 8, respectively. The statistical moments of the mentioned control variables are given in Table IV. The behavior of the statistical moments of these control variables is also similar to the statistical moments of the energy and security costs.

Moreover, the generating unit output powers are constant for all scenarios at buses 1, 2, 16, 18, 21 and 22. It shows that the optimal output power of these generating units is not sensitive to the uncertainties. The downward reserve at buses 7 and 21 are non-zero only for Case $\mathrm{C}$ with small probabilities of 0.0001 and 0.0003 , respectively. Therefore, this security control of these generating units is required only for a certain number of scenarios due to the superposition of both binary and continuous uncertainties. The downward reserve at buses 15 and 16 are non-zero for Case $\mathrm{A}$ and Case $\mathrm{C}$ with small probabilities of 0.00004 and 0.0008 , respectively. These results demonstrate that the downward reserves at these buses are useful only for the continuous uncertainties.
TABLE III.

STATISTICAL MOMENTS FOR ENERGY AND SECURITY COSTS FOR ALL CASES.

\begin{tabular}{clcccc}
\hline & & $\boldsymbol{\mu} \mathbf{( \$ )}$ & $\boldsymbol{\sigma}$ & $\boldsymbol{\gamma}$ & $\boldsymbol{\kappa}$ \\
\hline \multirow{3}{*}{ security cost } & Only Continuous (Case A) & 3.4785 & 4.7372 & 1.5254 & 4.9515 \\
& Only Binary (Case B) & 0.5483 & 5.0949 & 11.3729 & 154.9106 \\
& Both of them (Case C) & 3.9763 & 6.7355 & 4.7583 & 45.3816 \\
\hline \multirow{3}{*}{ energy cost } & Only Continuous (Case A) & 1334.17 & 2.1985 & 3.3447 & 13.9087 \\
& Only Binary (Case B) & 1333.90 & 4.6438 & 16.3245 & 346.0851 \\
& Both of them (Case C) & 1334.64 & 5.0752 & 12.4227 & 237.2512 \\
\hline
\end{tabular}

TABLE IV.

STATISTICAL MOMENTS OF SEVERAL CONTROL VARIABLES FOR ALL CASES.

\begin{tabular}{clcccc}
\hline & & $\boldsymbol{\mu}(\mathbf{M W})$ & $\boldsymbol{\sigma}$ & $\boldsymbol{\gamma}$ & $\boldsymbol{\kappa}$ \\
\hline $\begin{array}{c}\text { generating unit } \\
\text { output power }\end{array}$ & Only Continuous (Case A) & 155.536 & 1.7062 & 3.5607 & 15.6474 \\
at bus 15 & Only Binary (Case B) & 155.021 & 0.4589 & 37.0299 & 1571.15 \\
\hline \multirow{2}{*}{$\begin{array}{l}\text { Bpward reserve } \\
\text { at bus 13 }\end{array}$} & Only Continuous (Case A) & 16.7006 & 20.6643 & 0.9273 & 2.5657 \\
& Only Binary (Case B) & 2.0749 & 20.7900 & 12.7593 & 185.4019 \\
downward & Both of them (Case C) & 18.4570 & 28.2889 & 4.5386 & 44.7256 \\
reserve & Only Continuous (Case A) & 19.5700 & 26.9786 & 1.54507 & 5.1106 \\
at bus 22 & Only Binary (Case B) & 1.24447 & 16.3213 & 14.9583 & 238.6915 \\
\hline
\end{tabular}




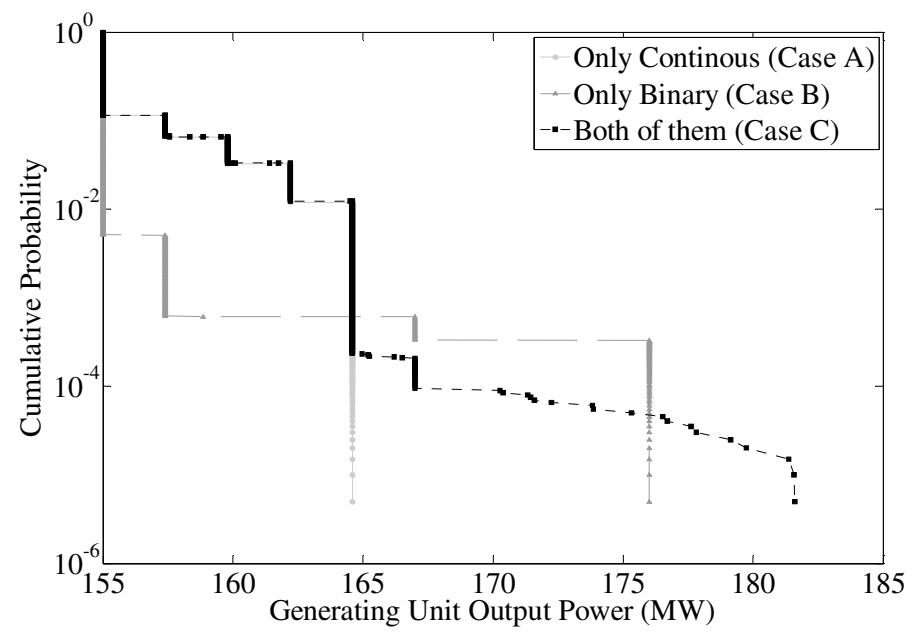

Fig. 6. Cumulative distribution of the generating unit output power at bus 15 for all cases.

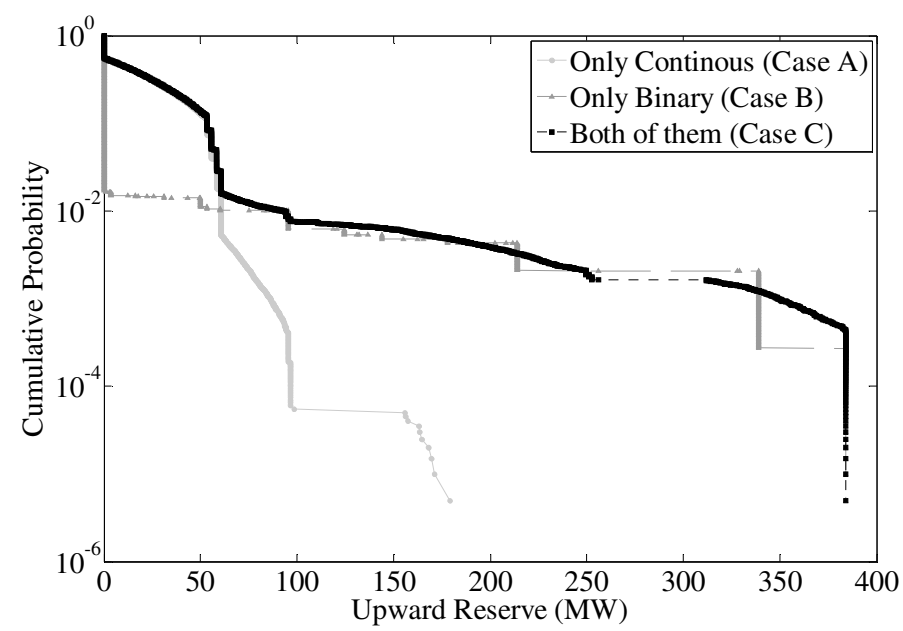

Fig. 7. Cumulative distribution of the upward reserve at bus 13 for all cases.

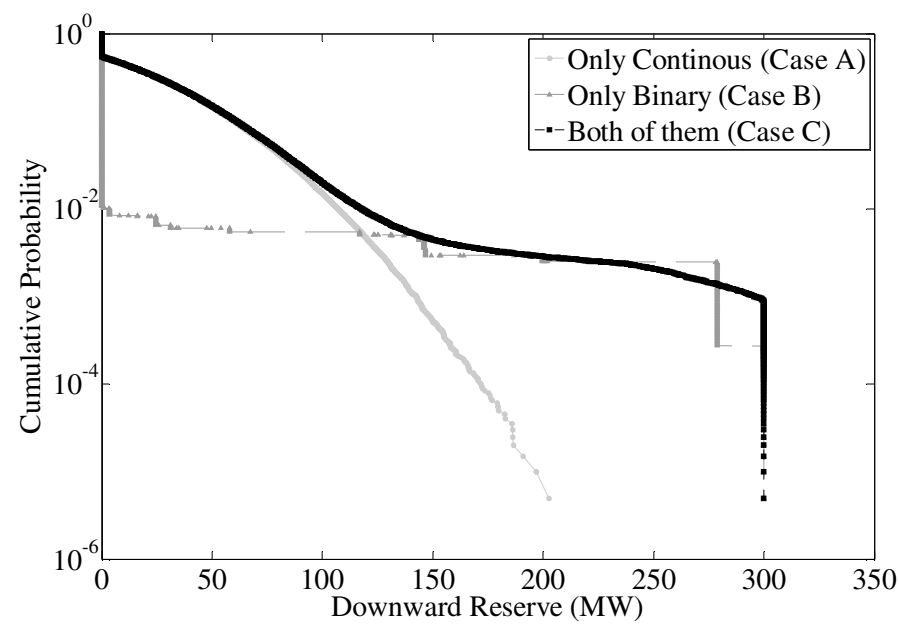

Fig. 8. Cumulative distribution of the downward reserve at bus 22 for all cases.

\section{CONCLUSIONS}

Different source of uncertainties are classified based on their binary and continuous attributes. The outage of generating units and transmission elements are considered as binary uncertainties and the forecast errors of loads and wind power outputs are assumed as continuous uncertainties. The model of wind power forecast errors is revisited in order to provide more realistic stochastic scenarios. Then, an optimization approach is developed to investigate the impact of different source of uncertainties on the cost of energy and security controls. The main advantage of the proposed optimization is that the effect of stochastic scenarios, as many as required, can be evaluated without increasing the size of the optimization problem. The stochastic scenarios are provided using a MCS. The probability distributions and statistical moments of the optimization outcomes, including objective functions and control variables, are then evaluated. The simulation results demonstrated that the binary uncertainties lead to higher costs with small probabilities, whereas, the continuous uncertainties results in lower costs with high probability. This conclusion is useful for the management of power systems with high penetration of intermittent renewable energy resources. Furthermore, the fact that the proposed method addresses a time instant and it does not take into account the inter-temporal constraints (e.g. startup/shut down and ramp up/ramp down) additional investigation is required to extend this method for the multiperiod problem.

\section{REFERENCES}

[1] J. Wang, N. E. Redondo, F. D. Galiana, "Demand-Side Reserve Offers in Joint Energy/Reserve Electricity Markets," IEEE Trans. Power Syst., vol. 18, no. 4, pp. 1300 - 1306, 2003.

[2] R. Billinton, R. N. Allan, Reliability Evaluation of Power Systems, Springer, 1996.

[3] A. J. Conejo, M. Carrión, M. J. Morales, Decision Making Under Uncertainty in Electricity Markets, Springer, 2010.

[4] L. Mili, "Taxonomy of the Characteristics of Power System Operating States," in Proc. of $2^{\text {nd }}$ NSF-RESIN workshop, 2011.

[5] O. Alizadeh Mousavi, R. Cherkaoui, "Discussion on Uncertainty Management in Power Systems," in IREP Symp. - Bulk Power System Dynamics and Control IX (IREP), Rethymnon, 2013.

[6] W. Li, R. Billinton, Reliability Assessment of Electrical Power Systems Using Monte Carlo Methods, Springer, 1994.

[7] B.-M. Hodge, D. Lew, M. Milligan, H. Holttinen, S. Sillanpää, E. Gómez-Lázaro, R. Scharff, L. Söder, X. Larsén, G. Giebel, D. Flynn, J. Dobschinski, "Wind Power Forecasting Error Distributions: An International Comparison," in $11^{\text {th }}$ Annu. Int. Workshop on Large-Scale Integration of Wind Power into Power Systems as well as on Transmission Networks for Offshore Wind Power Plants Conf., Lisbon, 2012.

[8] K. Dietrich, J. Latorre, L. Olmos, A. Ramos, I. Perez-Arriaga, "Stochastic unit commitment considering uncertain wind production in an isolated system," in $4^{\text {th }}$ Conf. Energy Economics and Technology, Dresden, 2009.

[9] H. Bludzuweit, J. Dominguez-Navarro, A. Llombart, "Statistical analysis of wind power forecast error," IEEE Trans. Power Syst., vol. 23, no. 3, pp. 983 - 991, 2008.

[10] "MathWorks - Pearson system random numbers," 2013. [Online]. Available: http://www.mathworks.ch/ch/help/stats/pearsrnd.html.

[11] "Gurobi Optimization," 2013. [Online]. Available: http://www.gurobi.com/.

[12] J. Lofberg, "YALMIP : a toolbox for modeling and optimization in MATLAB," in IEEE Int. Symp. Computer Aided Control Systems Design, Taipei, 2004.

[13] "The IEEE Reliability Test System - 1996," IEEE Trans. Power Syst., vol. 14, no. 3, pp. 1010-1020, 1999 